\title{
Lucas Polynomial Approach for System of High-Order Linear Differential Equations and Residual Error Estimation
}

\author{
Muhammed Çetin, ${ }^{1}$ Mehmet Sezer, ${ }^{1}$ and Coşkun Güler ${ }^{2}$ \\ ${ }^{1}$ Department of Mathematics, Faculty of Science, Celal Bayar University, Manisa, Turkey \\ ${ }^{2}$ Department of Mathematical Engineering, Faculty of Chemistry-Metallurgical, Yıldız Technical University, Istanbul, Turkey \\ Correspondence should be addressed to Mehmet Sezer; mehmet.sezer@cbu.edu.tr
}

Received 15 November 2014; Accepted 5 January 2015

Academic Editor: Mingshu Peng

Copyright (c) 2015 Muhammed Çetin et al. This is an open access article distributed under the Creative Commons Attribution License, which permits unrestricted use, distribution, and reproduction in any medium, provided the original work is properly cited.

\begin{abstract}
An approximation method based on Lucas polynomials is presented for the solution of the system of high-order linear differential equations with variable coefficients under the mixed conditions. This method transforms the system of ordinary differential equations (ODEs) to the linear algebraic equations system by expanding the approximate solutions in terms of the Lucas polynomials with unknown coefficients and by using the matrix operations and collocation points. In addition, the error analysis based on residual function is developed for present method. To demonstrate the efficiency and accuracy of the method, numerical examples are given with the help of computer programmes written in Maple and Matlab.
\end{abstract}

\section{Introduction}

The systems of differential equations with variable coefficients have been encountered in many scientific and technological problems. Some of these differential equation systems do not have analytic solutions, so numerical methods are required. The systems of linear differential equations have been solved by many mathematicians and engineers by using the various methods such as variational iteration method [1], the differential transform method [2-5], the Adomian decomposition method $[6,7]$ and the linearizability criteria $[8,9]$, finite difference method [10], and Adomian-Pade technique [11].

Taylor, Chebyshev, Legendre, Berstein, Hermite, Laguerre, and Bessel matrix methods are used for solving differential and integral equations, integrodifferential-difference equations, and their systems in [12-20]. In this paper, by means of the above-mentioned methods and the Lucas polynomials, we have developed a new method called Lucas collocation method to solve the system of high-order linear differential equations with variable coefficients in the form

$$
\begin{array}{r}
L\left[y_{i}(x)\right]=\sum_{n=0 j=1}^{m} \sum_{j=1}^{k} p_{i, j}^{n}(x) y_{j}^{(n)}(x)=g_{i}(x), \\
(i=1,2, \ldots, k, 0 \leq a \leq x \leq b)
\end{array}
$$

under the mixed conditions

$$
\begin{aligned}
& \sum_{n=0}^{m-1}\left[a_{i, n}^{j} y_{j}^{(n)}(a)+b_{i, n}^{j} y_{j}^{(n)}(b)\right]=c_{j, i}, \\
& (i=0,1, \ldots, m-1, j=1,2, \ldots, k, n=0,1, \ldots, m-1),
\end{aligned}
$$

where $y_{j}^{(0)}(x)=y_{j}(x)$ is an unknown function, $p_{i, j}^{n}(x)$ and $g_{i}(x)$ are the known continuous functions defined on interval $[a, b]$, and coefficients $a_{i, n}^{j}, b_{i, n}^{j}$ and $c_{j, i}$ are the real constants.

In addition, by improving the present method with the help of the residual error function used in [21-25], we obtain the corrected approximate solutions of the system (1) expressed in the truncated Lucas series form

$$
y_{j, N, M}(x)=y_{j, N}(x)+e_{j, N, M}(x), \quad j=1,2, \ldots, k,
$$

where

$$
y_{j} \cong y_{j, N}(x)=\sum_{n=0}^{N} a_{j, n} L_{n}(x)
$$

is the Lucas polynomial solution and

$$
e_{j, N, M}(x)=\sum_{n=0}^{M} a_{j, n}^{*} L_{n}(x), \quad(M>N)
$$


is the solution of the error problem obtained with the aid of the residual error function. Here $a_{j, n},(n=0,1,2, \ldots, N)$ and $a_{j, n}^{*},(n=0,1,2, \ldots, N)$ are the unknown Lucas coefficients, and $L_{n}(x), n=0,1, \ldots, N$ are the Lucas polynomials defined by

$$
\begin{gathered}
L_{0}(x)=2 ; \\
L_{n}(x)=\sum_{k=0}^{[n / 2]} \frac{n}{n-k}\left(\begin{array}{c}
n-k \\
k
\end{array}\right) x^{n-2 k}, \quad(n \geq 1), \\
{\left[\frac{n}{2}\right]= \begin{cases}\frac{n}{2}, & n \text { even } \\
\frac{(n-1)}{2}, & n \text { odd }\end{cases} }
\end{gathered}
$$

[26-28]. The purpose of this study is to improve the approximate solutions for high-order systems of ODEs by means of the residual error function and to give an efficient and useful error estimation via the error problem.
In order to find solutions of the system (1), with the mixed conditions (2), we can use the collocation points defined by

$$
x_{i}=a+\frac{b-a}{N} i, \quad i=0,1, \ldots, N, 0 \leq a \leq x \leq b .
$$

\section{Fundamental Matrix Relations}

The Lucas polynomials $L_{n}(x)$ can be written in the matrix form as

$$
\mathbf{L}(x)=\mathbf{X}(x) \mathbf{D}^{T}
$$

where

$$
\begin{aligned}
\mathbf{L}(x)= & {\left[\begin{array}{lllll}
L_{0}(x) & L_{1}(x) & L_{2}(x) & \cdots & L_{N}(x)
\end{array}\right] } \\
& \mathbf{X}(x)=\left[\begin{array}{lllll}
1 & x & x^{2} & \cdots & x^{N}
\end{array}\right]
\end{aligned}
$$

and if $N$ is odd,

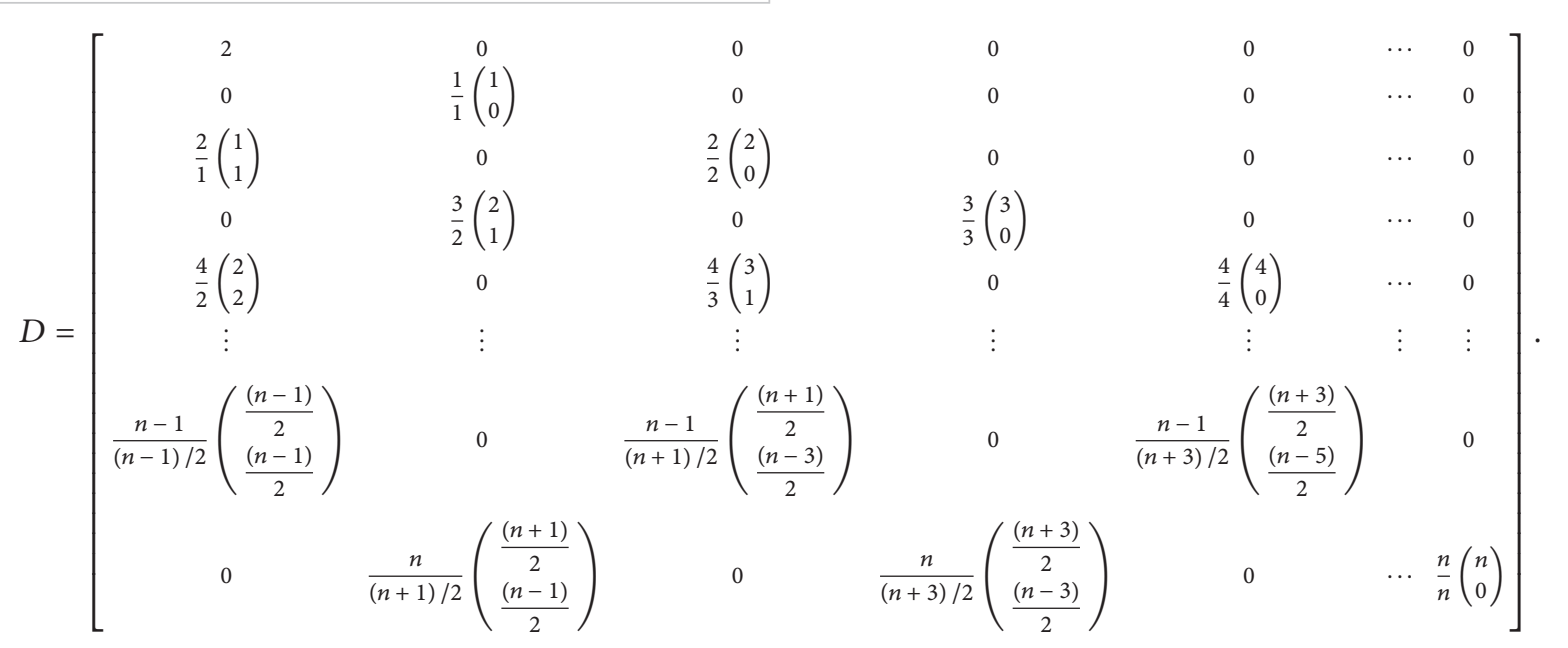

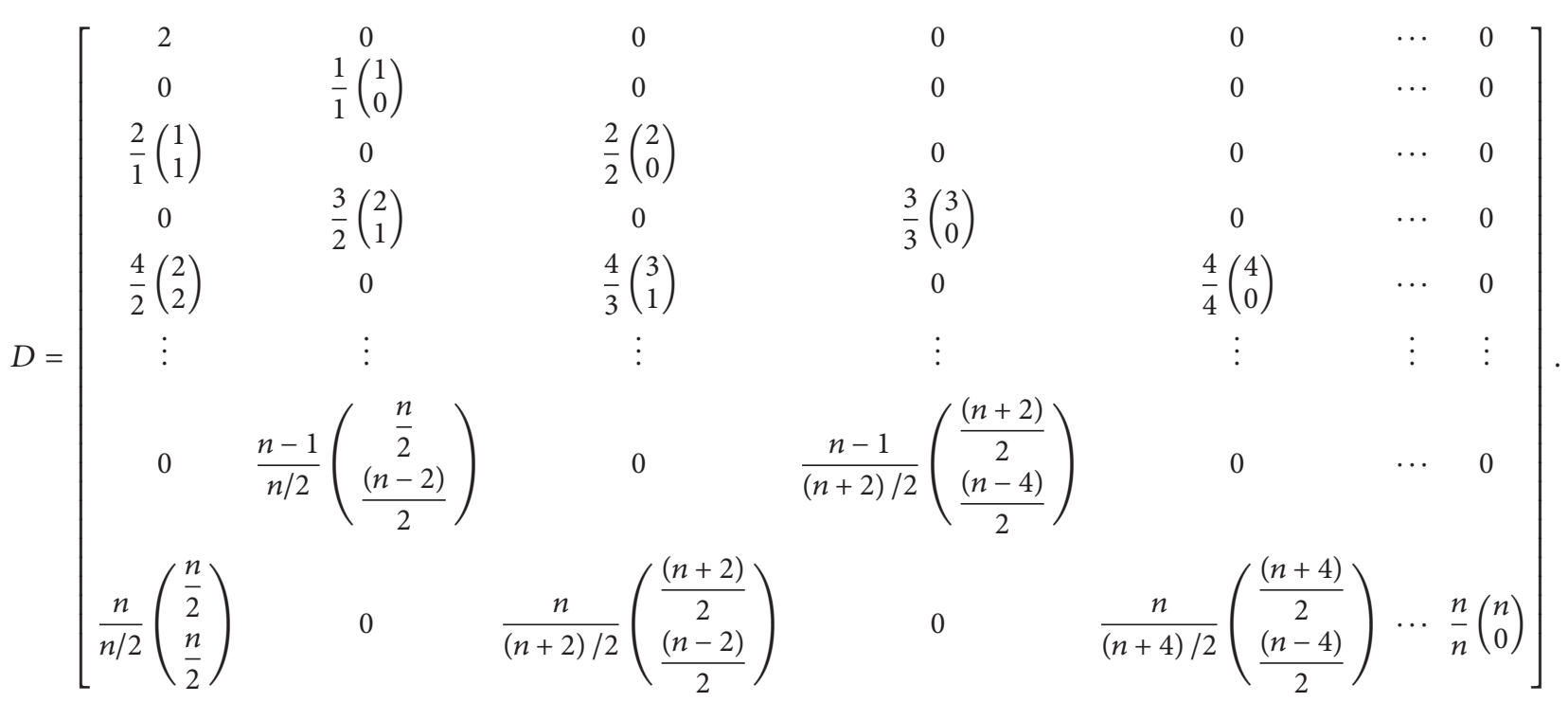


We can write the approximate solutions $y_{j, N}(x)$ given by (4) in the matrix form

$$
y_{j, N}(x)=\mathbf{L}(x) \mathbf{A}_{j}, \quad j=1,2, \ldots, k,
$$

where

$$
\mathbf{A}_{j}=\left[\begin{array}{lllll}
a_{j, 0} & a_{j, 1} & a_{j, 2} & \cdots & a_{j, N}
\end{array}\right]^{T} .
$$

From (8) and (12), we obtain the matrix relation

$$
y_{j, N}(x)=\mathbf{X}(x) \mathbf{D}^{T} \mathbf{A}_{j} .
$$

Also, the relation between the matrix $\mathbf{X}(x)$ and its derivatives $\mathbf{X}^{(k)}(x)$ is

$$
\mathbf{X}^{(k)}(x)=\mathbf{X}(x) \mathbf{B}^{k},
$$

where

$$
\mathbf{B}=\left[\begin{array}{ccccc}
0 & 1 & 0 & \cdots & 0 \\
0 & 0 & 2 & \cdots & 0 \\
\vdots & \vdots & \vdots & \ddots & \vdots \\
0 & 0 & 0 & \cdots & N \\
0 & 0 & 0 & \cdots & 0
\end{array}\right]
$$

and $\mathbf{B}^{0}=\mathbf{I}_{(N+1) \times(N+1)}$ is the unit matrix.

By using the relations (14) and (15), we obtain the following relations:

$$
\begin{aligned}
& y_{j, N}^{(i)}(x)=\mathbf{X}(x) \mathbf{B}^{i} \mathbf{D}^{T} \mathbf{A}_{j}, \\
& i=0,1, \ldots, m, \quad j=1,2, \ldots, k .
\end{aligned}
$$

Hence, we can write the matrix relations as

$$
\mathbf{Y}^{(i)}(x)=\overline{\mathbf{X}}(x)(\overline{\mathbf{B}})^{i} \overline{\mathbf{D}} \mathbf{A}, \quad i=0,1, \ldots, m,
$$

where

$$
\begin{aligned}
& \mathbf{Y}^{(i)}(x)=\left[\begin{array}{c}
y_{1, N}^{(i)}(x) \\
y_{2, N}^{(i)}(x) \\
\vdots \\
y_{k, N}^{(i)}(x)
\end{array}\right], \\
& \overline{\mathbf{X}}(x)=\left[\begin{array}{cccc}
\mathbf{X}(x) & 0 & \cdots & 0 \\
0 & \mathbf{X}(x) & \cdots & 0 \\
\vdots & \vdots & \ddots & \vdots \\
0 & 0 & \cdots & \mathbf{X}(x)
\end{array}\right] \text {, } \\
& \overline{\mathbf{B}}=\left[\begin{array}{cccc}
\mathbf{B} & 0 & \cdots & 0 \\
0 & \mathbf{B} & \cdots & 0 \\
\vdots & \vdots & \ddots & \vdots \\
0 & 0 & \cdots & \mathbf{B}
\end{array}\right] \\
& \overline{\mathbf{D}}=\left[\begin{array}{cccc}
\mathbf{D}^{T} & 0 & \cdots & 0 \\
0 & \mathbf{D}^{T} & \cdots & 0 \\
\vdots & \vdots & \ddots & \vdots \\
0 & 0 & \cdots & \mathbf{D}^{T}
\end{array}\right], \quad \mathbf{A}=\left[\begin{array}{c}
\mathbf{A}_{1} \\
\mathbf{A}_{2} \\
\vdots \\
\mathbf{A}_{k}
\end{array}\right]
\end{aligned}
$$

\section{Method for Solution}

Firstly, we can write the system (1) in the matrix form

$$
\sum_{i=0}^{m} \mathbf{P}_{i}(x) \mathbf{Y}^{(i)}(x)=\mathbf{G}(x)
$$

where

$$
\mathbf{P}_{i}(x)=\left[\begin{array}{cccc}
p_{1,1}^{i}(x) & p_{1,2}^{i}(x) & \cdots & p_{1, k}^{i}(x) \\
p_{2,1}^{i}(x) & p_{2,2}^{i}(x) & \cdots & p_{2, k}^{i}(x) \\
\vdots & \vdots & \ddots & \vdots \\
p_{k, 1}^{i}(x) & p_{k, 2}^{i}(x) & \cdots & p_{k, k}^{i}(x)
\end{array}\right],
$$

$$
\mathbf{Y}^{(i)}(x)=\left[\begin{array}{c}
y_{1, N}^{(i)}(x) \\
y_{2, N}^{(i)}(x) \\
\vdots \\
y_{k, N}^{(i)}(x)
\end{array}\right], \quad \mathbf{G}(x)=\left[\begin{array}{c}
g_{1}(x) \\
g_{2}(x) \\
\vdots \\
g_{k}(x)
\end{array}\right] .
$$

By substituting the collocation points (7) into (20) we obtain the system of matrix equations

$$
\sum_{i=0}^{m} \mathbf{P}_{i}\left(x_{s}\right) \mathbf{Y}^{(i)}\left(x_{s}\right)=\mathbf{G}\left(x_{s}\right), \quad s=0,1, \ldots, N,
$$

or the compact form

$$
\sum_{i=0}^{m} \mathbf{P}_{i} \mathbf{Y}^{(i)}=\mathbf{G}
$$

where

$$
\begin{aligned}
& \mathbf{P}_{i}=\left[\begin{array}{cccc}
\mathbf{P}_{i}\left(x_{0}\right) & 0 & \cdots & 0 \\
0 & \mathbf{P}_{i}\left(x_{1}\right) & \cdots & 0 \\
\vdots & \vdots & \ddots & \vdots \\
0 & 0 & \cdots & \mathbf{P}_{i}\left(x_{N}\right)
\end{array}\right], \\
& \mathbf{Y}^{(i)}=\left[\begin{array}{c}
\mathbf{Y}^{(i)}\left(x_{0}\right) \\
\mathbf{Y}^{(i)}\left(x_{1}\right) \\
\vdots \\
\mathbf{Y}^{(i)}\left(x_{N}\right)
\end{array}\right], \\
& \mathbf{G}=\left[\begin{array}{c}
\mathbf{G}\left(x_{0}\right) \\
\mathbf{G}\left(x_{1}\right) \\
\vdots \\
\mathbf{G}\left(x_{N}\right)
\end{array}\right] .
\end{aligned}
$$

From the relation (18) and the collocation points (7), we have

$$
\mathbf{Y}^{(i)}\left(x_{s}\right)=\overline{\mathbf{X}}\left(x_{s}\right)(\overline{\mathbf{B}})^{i} \overline{\mathbf{D}} \mathbf{A}, \quad s=0,1, \ldots, N
$$

or, briefly,

$$
\mathbf{Y}^{(i)}=\mathbf{X}(\overline{\mathbf{B}})^{i} \overline{\mathbf{D}} \mathbf{A},
$$


where

$$
\begin{gathered}
\mathbf{X}=\left[\begin{array}{c}
\overline{\mathbf{X}}\left(x_{0}\right) \\
\overline{\mathbf{X}}\left(x_{1}\right) \\
\vdots \\
\overline{\mathbf{X}}\left(x_{N}\right)
\end{array}\right], \\
\overline{\mathbf{X}}\left(x_{s}\right)=\left[\begin{array}{cccc}
\mathbf{X}\left(x_{s}\right) & 0 & \cdots & 0 \\
0 & \mathbf{X}\left(x_{s}\right) & \cdots & 0 \\
\vdots & \vdots & \ddots & \vdots \\
0 & 0 & \cdots & \mathbf{X}\left(x_{s}\right)
\end{array}\right] .
\end{gathered}
$$

By substituting (26) into (23), we obtain the fundamental matrix equation as

$$
\left\{\sum_{i=0}^{m} \mathbf{P}_{i} \mathbf{X}(\overline{\mathbf{B}})^{i} \overline{\mathbf{D}}\right\} \mathbf{A}=\mathbf{G}
$$

In (28) the full dimensions of the matrices $\mathbf{P}_{i}, \mathbf{X}, \overline{\mathbf{B}}, \overline{\mathbf{D}}$, $\mathbf{A}$, and $\mathbf{G}$ are $k(N+1) \times k(N+1), k(N+1) \times k(N+1)$, $k(N+1) \times k(N+1), k(N+1) \times k(N+1), k(N+1) \times 1$, and $k(N+1) \times 1$, respectively.

The fundamental matrix equation (28) corresponding to (1) can be written in the form

$$
\mathbf{W A}=\mathbf{G} \quad \text { or } \quad[\mathbf{W} ; \mathbf{G}] .
$$

This is a linear system of $k(N+1)$ algebraic equations in the $k(N+1)$ unknown Lucas coefficients such that

$$
\begin{array}{r}
\mathbf{W}=\sum_{i=0}^{m} \mathbf{P}_{i} \mathbf{X}(\overline{\mathbf{B}})^{i} \overline{\mathbf{D}}=\left[w_{p, q}\right], \\
p, q=1,2, \ldots, k(N+1) .
\end{array}
$$

By using the conditions (2) and the relations (18), the matrix form for the conditions is obtained as

$$
\sum_{j=0}^{m-1}\left[a_{j} \overline{\mathbf{X}}(a)+b_{j} \overline{\mathbf{X}}(b)\right](\overline{\mathbf{B}})^{j} \overline{\mathbf{D}} \mathbf{A}=\mathbf{C},
$$

where

$$
\begin{gathered}
a_{j}=\left[\begin{array}{cccc}
a_{j}^{1} & 0 & \cdots & 0 \\
0 & a_{j}^{2} & \cdots & 0 \\
\vdots & \vdots & \ddots & \vdots \\
0 & 0 & \cdots & a_{j}^{k}
\end{array}\right], \\
b_{j}=\left[\begin{array}{cccc}
b_{j}^{1} & 0 & \cdots & 0 \\
0 & b_{j}^{2} & \cdots & 0 \\
\vdots & \vdots & \ddots & \vdots \\
0 & 0 & \cdots & b_{j}^{k}
\end{array}\right], \quad \mathbf{C}=\left[\begin{array}{c}
c_{1} \\
c_{2} \\
\vdots \\
c_{k}
\end{array}\right],
\end{gathered}
$$

$$
\begin{array}{r}
a_{j}^{i}=\left[\begin{array}{c}
a_{0, j}^{i} \\
a_{1, j}^{i} \\
\vdots \\
a_{m-1, j}^{i}
\end{array}\right], \quad b_{j}^{i}=\left[\begin{array}{c}
b_{0, j}^{i} \\
b_{1, j}^{i} \\
\vdots \\
b_{m-1, j}^{i}
\end{array}\right], \quad c_{i}=\left[\begin{array}{c}
c_{i, 0} \\
c_{i, 1} \\
\vdots \\
c_{i, m-1}
\end{array}\right], \\
i=0,1, \ldots, k .
\end{array}
$$

Hence, the fundamental matrix form for conditions is

$$
\mathbf{U A}=\mathbf{C} \quad \text { or } \quad[\mathbf{U} ; \mathbf{C}]
$$

such that

$$
\mathbf{U}=\sum_{j=0}^{m-1}\left[a_{j} \overline{\mathbf{X}}(a)+b_{j} \overline{\mathbf{X}}(b)\right](\overline{\mathbf{B}})^{j} \overline{\mathbf{D}} .
$$

Consequently, by replacing the row matrices (33) by last rows of the matrix (29), we obtain the new augmented matrix

$$
[\widetilde{\mathbf{W}} ; \widetilde{\mathbf{G}}] \text {. }
$$

We do not have to change the last rows of the matrix equation given by (29). If the matrix $\widetilde{W}$ is singular, then rows of the matrix (33) can be replaced with any rows of the matrix (29). If $\operatorname{rank} \widetilde{\mathbf{W}}=\operatorname{rank}[\widetilde{\mathbf{W}} ; \widetilde{\mathbf{G}}]=N+1$, then we can write

$$
\mathbf{A}=(\widetilde{\mathbf{W}})^{-1} \widetilde{\mathbf{G}} \text {. }
$$

Hence, the unknown Lucas coefficients matrix $\mathbf{A}$ is determined. We can find the Lucas polynomial solutions

$$
y_{j, N}(x)=\sum_{n=0}^{N} a_{j, n} L_{n}(x), \quad j=1,2, \ldots, k .
$$

\section{Residual Correction and Error Estimation}

In this section, we will give an error estimation for the Lucas polynomial solutions (4) with the residual error function [2125]. Moreover, we will improve the solution (4) by means of the residual error function. Firstly, we can define the residual function of the method as

$$
\begin{aligned}
& R_{i, N}(x)=L\left[y_{j, N}(x)\right]-g_{i}(x), \\
& (i=1,2, \ldots, k, j=1,2, \ldots, k) .
\end{aligned}
$$

Here, $y_{j, N}(x)$ represent the Lucas polynomial solutions given by (4) of the problem (1) and (2). Hence, $y_{j, N}(x)$ satisfies the problem

$$
\begin{aligned}
& L\left[y_{j, N}(x)\right]=\sum_{n=0}^{m} \sum_{j=1}^{k} p_{i, j}^{n}(x) y_{j, N}^{(n)}(x)=g_{i}(x)+R_{i, N}(x), \\
& (i=1,2, \ldots, k, j=1,2, \ldots, k, n=0,1, \ldots, m), \\
& \sum_{n=0}^{m-1}\left[a_{i, n}^{j} y_{j, N}^{(n)}(a)+b_{i, n}^{j} y_{j, N}^{(n)}(b)\right]=c_{j, i}, \\
& (i=0,1, \ldots, m-1, j=1,2, \ldots, k, n=0,1, \ldots, m-1) .
\end{aligned}
$$


Also, the error function $e_{j, N}(x)$ can be defined as

$$
e_{j, N}(x)=y_{j}(x)-y_{j, N}(x),
$$

where $y_{j}(x)$ are the exact solutions of the problem (1) and (2). From (1), (2), (38), and (40), we obtain the error differential equation system

$$
L\left[e_{j, N}(x)\right]=L\left[y_{j}(x)\right]-L\left[y_{j, N}(x)\right]=-R_{i, N}(x)
$$

with the homogeneous mixed conditions

$$
\begin{aligned}
& \sum_{n=0}^{m-1}\left[a_{i, n}^{j} e_{j, N}^{(n)}(a)+b_{i, n}^{j} e_{j, N}^{(n)}(b)\right]=0, \\
& (i=0,1, \ldots, m-1, j=1,2, \ldots, k, n=0,1, \ldots, m-1)
\end{aligned}
$$

or, openly, the error problem

$$
\begin{aligned}
& \sum_{n=0}^{m} \sum_{j=1}^{k} p_{i, j}^{n}(x) e_{j, N}^{(n)}(x)=-R_{i, N}(x), \\
& (i=1,2, \ldots, k, j=1,2, \ldots, k, n=0,1, \ldots, m), \\
& \sum_{n=0}^{m-1}\left[a_{i, n}^{j} e_{j, N}^{(n)}(a)+b_{i, n}^{j} e_{j, N}^{(n)}(b)\right]=0, \\
& (i=0,1, \ldots, m-1, j=1,2, \ldots, k, n=0,1, \ldots, m-1) .
\end{aligned}
$$

Here, note that the nonhomogeneous mixed conditions

$$
\begin{aligned}
& \sum_{n=0}^{m-1}\left[a_{i, n}^{j} y_{j}^{(n)}(a)+b_{i, n}^{j} y_{j}^{(n)}(b)\right]=c_{j, i}, \\
& (i=0,1, \ldots, m-1, j=1,2, \ldots, k, n=0,1, \ldots, m-1), \\
& \sum_{n=0}^{m-1}\left[a_{i, n}^{j} y_{j, N}^{(n)}(a)+b_{i, n}^{j} y_{j, N}^{(n)}(b)\right]=c_{j, i}, \\
& (i=0,1, \ldots, m-1, j=1,2, \ldots, k, n=0,1, \ldots, m-1)
\end{aligned}
$$

are reduced to homogeneous mixed conditions

$$
\begin{aligned}
& \sum_{n=0}^{m-1}\left[a_{i, n}^{j} e_{j, N}^{(n)}(a)+b_{i, n}^{j} e_{j, N}^{(n)}(b)\right]=0, \\
& (i=0,1, \ldots, m-1, j=1,2, \ldots, k, n=0,1, \ldots, m-1) .
\end{aligned}
$$

The error problem (43) can be solved by using the procedure in Section 3. Thus, we obtain the approximation $e_{j, N, M}(x)$ to $e_{j, N}(x)$ as follows:

$$
e_{j, N, M}(x)=\sum_{n=0}^{M} a_{j, n}^{*} L_{n}(x), \quad(M>N, j=1,2, \ldots, k) .
$$

Consequently, the corrected Lucas polynomial solution $y_{j, N, M}(x)=y_{j, N}(x)+e_{j, N, M}(x)$ is obtained by means of the polynomials $y_{j, N}(x)$ and $e_{j, N, M}(x), M>N$. Also, we construct the error function $e_{j, N}(x)=y_{j}(x)-y_{j, N}(x)$, the estimated error function $e_{j, N, M}(x)$, and the corrected error function $E_{j, N, M}(x)=e_{j, N}(x)-e_{j, N, M}(x)=y_{j}(x)-y_{j, N, M}(x)$.

\section{Numerical Examples}

In this section, the several numerical examples are given to demonstrate the efficiency and applicability of our method. The computations related to the examples are calculated by using a computer programme which is called Maple and the figures are drawn in Matlab. In tables and figures, we calculate the values of the Lucas polynomial solution $y_{j, N}(x)$, the corrected Lucas polynomial solution $y_{j, N, M}(x)=y_{j, N}(x)+$ $e_{j, N, M}(x)$, the actual absolute error function $\left|e_{j, N}(x)\right|=$ $\left|y_{j}(x)-y_{j, N}(x)\right|$, and the estimated absolute error function $\left|e_{j, N, M}(x)\right|$.

Example 1. Let us consider the system of second-order linear differential equations given by

$$
\begin{array}{r}
y_{1}^{(2)}(x)+x y_{1}(x)+x y_{2}(x)=2, \\
y_{2}^{(2)}(x)+2 x y_{2}(x)+2 x y_{1}(x)=-2, \\
0 \leq x \leq 1
\end{array}
$$

with the boundary conditions

$$
\begin{array}{ll}
y_{1}(0)=0, & y_{1}(1)=0, \\
y_{2}(0)=0, & y_{2}(1)=0
\end{array}
$$

which has the exact solutions $y_{1}(x)=x^{2}-x$ and $y_{2}(x)=$ $-x^{2}+x[30]$. In this problem $k=2, m=2, p_{1,1}^{2}=1, p_{1,1}^{1}=0$, $p_{1,1}^{0}=x, p_{1,2}^{2}=0, p_{1,2}^{1}=0, p_{1,2}^{0}=x, g_{1}(x)=2, p_{2,1}^{2}=0$, $p_{2,1}^{1}=0, p_{2,1}^{0}=2 x, p_{2,2}^{2}=1, p_{2,2}^{1}=0, p_{2,2}^{0}=2 x$, and $g_{2}(x)=$ -2 .

The approximate solutions $y_{1,2}(x)$ and $y_{2,2}(x)$ for $N=2$ are given by

$$
y_{i, 2}(x)=\sum_{n=0}^{2} a_{i, n} L_{n}(x), \quad(i=1,2) .
$$

The set of the collocation points given by (7) for $a=0$, $b=1$, and $N=2$ is calculated as

$$
\left\{x_{0}=0, x_{1}=\frac{1}{2}, x_{2}=1\right\} \text {. }
$$

From (28), the fundamental matrix equation of the problem (47) is written as

$$
\left\{\mathbf{P}_{0} \mathbf{X} \overline{\mathbf{D}}+\mathbf{P}_{1} \mathbf{X} \overline{\mathbf{B}} \overline{\mathbf{D}}+\mathbf{P}_{2} \mathbf{X}(\overline{\mathbf{B}})^{2} \overline{\mathbf{D}}\right\} \mathbf{A}=\mathbf{G} .
$$

By applying the procedure in Section 3, we obtain the Lucas polynomial solutions for $N=2$ as $y_{1,2}(x)=x^{2}-x$ and $y_{2,2}(x)=-x^{2}+x$, which are the exact solutions. 
TABLE 1: Numerical results of the exact solutions $y_{i}(x)$ and the approximate solutions $\left\{y_{i, N}(x), y_{i, N, M}(x)\right\}$ for $i=1,2, N=6$ and $M=8,11$ of problem (52).

\begin{tabular}{ccccc}
\hline$x_{i}$ & Exact solution & \multicolumn{3}{c}{ Lucas polynomial solutions } \\
& $y_{1}\left(x_{i}\right)$ & $y_{1,6}\left(x_{i}\right)$ & $y_{1,6,8}\left(x_{i}\right)$ & 0.806485719287 \\
\hline 0.1 & 0.806485719482 & 0.806485689143 & 0.625251707990 & 0.625251708032 \\
0.2 & 0.625251708173 & 0.625251667103 & 0.145975834235 & 0.145975834133 \\
0.5 & 0.145975834384 & 0.145975783291 & -0.252886020930 & -0.252886021210 \\
0.8 & -0.252886020789 & -0.252886103976 & -0.482526628764 & -0.482526626284 \\
1.0 & -0.482526627107 & -0.482526066408 & $y_{2,6,8}\left(x_{i}\right)$ & 0.098405441788 \\
\hline$x_{i}$ & $y_{2}\left(x_{i}\right)$ & $y_{2,6}\left(x_{i}\right)$ & 0.098405441810 & 0.193895101121 \\
0.1 & 0.098405441705 & 0.098405456828 & 0.193895101070 \\
0.2 & 0.193895101008 & 0.193895121487 & 0.466457257577 & 0.466457257606 \\
0.5 & 0.466457257480 & 0.466457282978 & 0.724228025625 & 0.724228025747 \\
0.8 & 0.724228025488 & 0.724228067006 & 0.890857246295 & 0.890857244962 \\
1.0 & 0.890857245275 & 0.890856966173 & & \\
\hline
\end{tabular}

TABLE 2: Comparison of the actual and estimated absolute errors for $N=6$ and $M=8,11$ of the problem (52).

\begin{tabular}{lcrr}
\hline$x_{i}$ & \multicolumn{2}{c}{ Actual absolute errors } & \multicolumn{2}{c}{ Estimated absolute errors } \\
\hline 0.1 & $\left|e_{1,6}\left(x_{i}\right)\right|=\left|y_{1}\left(x_{i}\right)-y_{1,6}\left(x_{i}\right)\right|$ & $\left|e_{1,6,8}\left(x_{i}\right)\right|$ & $3.0149 e-8$ \\
0.2 & $3.0339 e-8$ & $3.0144 e-8$ & $4.0930 e-8$ \\
0.5 & $4.1070 e-8$ & $4.0888 e-8$ & $5.0851 e-8$ \\
0.8 & $5.1093 e-8$ & $5.0951 e-8$ & $8.2796 e-8$ \\
1.0 & $8.3187 e-8$ & $8.3065 e-8$ & $5.5982 e-7$ \\
\hline$x_{i}$ & $5.6070 e-7$ & $5.6232 e-7$ & $\left|e_{2,6,11}\left(x_{i}\right)\right|$ \\
\hline 0.1 & $\left|e_{2,6}\left(x_{i}\right)\right|=\left|y_{2}\left(x_{i}\right)-y_{2,6}\left(x_{i}\right)\right|$ & $\left|e_{2,6,8}\left(x_{i}\right)\right|$ & $1.5040 e-8$ \\
0.2 & $1.5123 e-8$ & $1.5017 e-8$ & $2.0418 e-8$ \\
0.5 & $2.0479 e-8$ & $2.0367 e-8$ & $2.5377 e-8$ \\
0.8 & $2.5498 e-8$ & $2.5404 e-8$ & $4.1270 e-8$ \\
1.0 & $4.1518 e-8$ & $4.1390 e-8$ & $2.7877 e-7$ \\
\hline
\end{tabular}

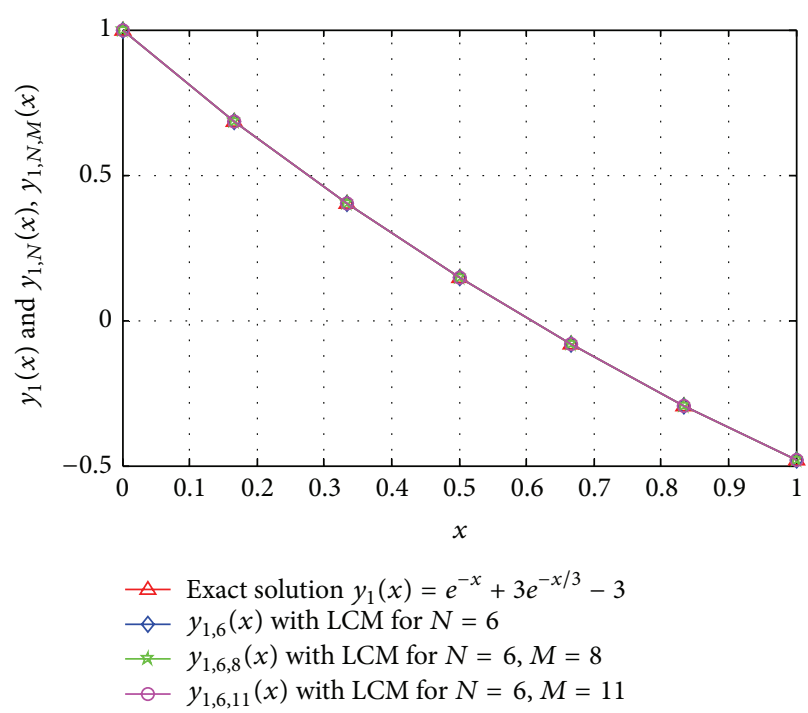

(a)

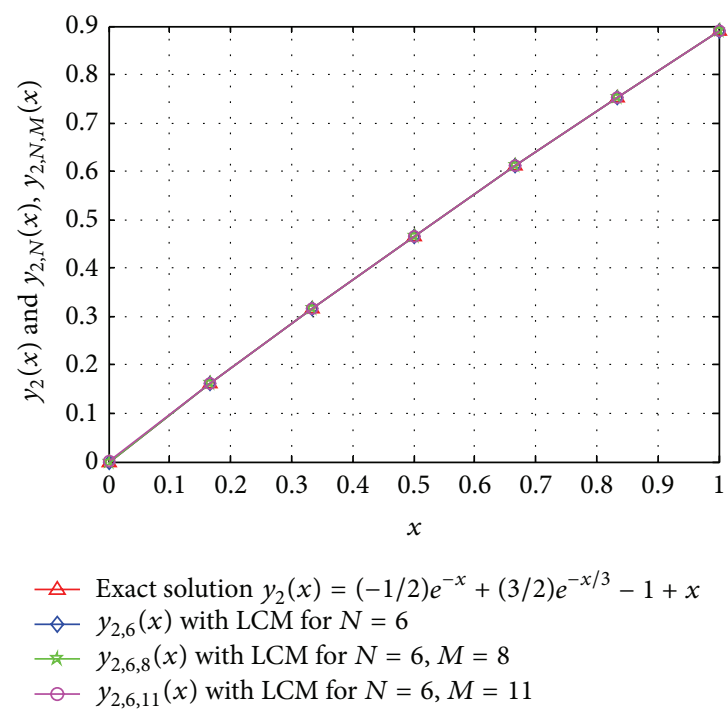

(b)

FIGURE 1: (a) Comparison of the exact solution $y_{1}(x)$ and the approximate solutions $y_{1, N}(x)$ and $y_{1, N, M}(x)$. (b) Comparison of the exact solution $y_{2}(x)$ and the approximate solutions $y_{2, N}(x)$ and $y_{2, N, M}(x)$. 


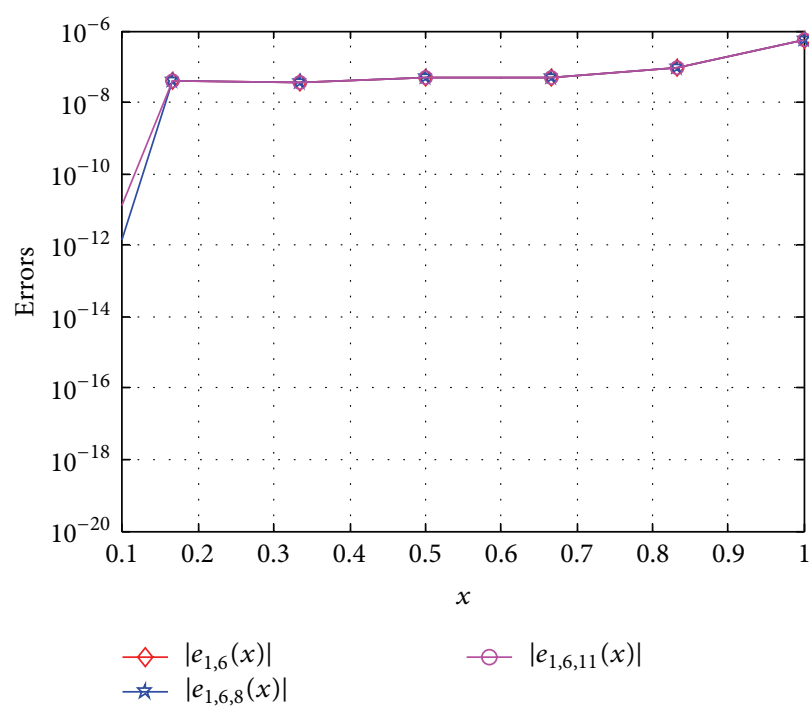

(a)

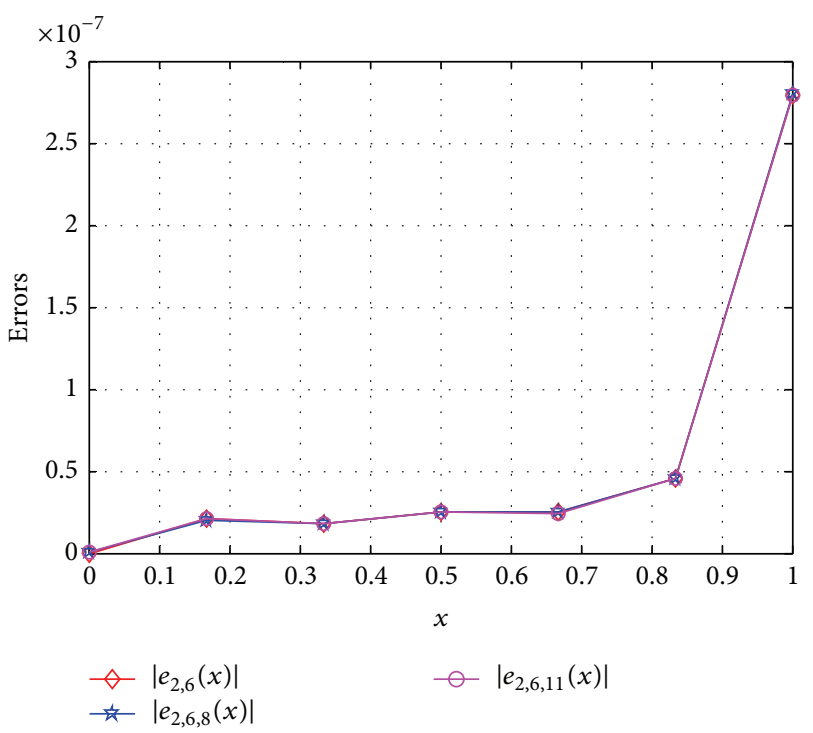

(b)

FIGURE 2: (a) Comparison of the actual and estimated absolute errors for $y_{1}(x)$. (b) Comparison of the actual and estimated absolute errors for $y_{2}(x)$.

TABLE 3: Numerical results of the exact solutions $y_{1}(x), y_{2}(x)$ and the Lucas polynomial solutions $y_{1, N}(x), y_{2, N}(x)$ for $N=6,8,11$ of problem (52).

\begin{tabular}{lcccc}
\hline$x_{i}$ & Exact solution & \multicolumn{3}{c}{ Lucas polynomial solutions } \\
& $y_{1}\left(x_{i}\right)$ & $y_{1,6}\left(x_{i}\right)$ & $y_{1,8}\left(x_{i}\right)$ & 0.806485719432 \\
0.1 & 0.806485719482 & 0.806485689143 & 0.625251708124 & 0.806485719479 \\
0.2 & 0.625251708173 & 0.625251667103 & 0.145975834317 & 0.625251708169 \\
0.5 & 0.145975834384 & 0.145975783291 & -0.252886020880 & 0.145975834379 \\
0.8 & -0.252886020789 & -0.252886103976 & -0.482526625954 & -0.252886020798 \\
1.0 & -0.482526627107 & -0.482526066408 & $y_{2,8}\left(x_{i}\right)$ & $y_{2,11}\left(x_{i}\right)$ \\
\hline$x_{i}$ & $y_{2}\left(x_{i}\right)$ & $y_{2,6}\left(x_{i}\right)$ & 0.098405441730 & 0.098405441706 \\
\hline 0.1 & 0.098405441705 & 0.098405456828 & 0.193895101033 & 0.193895101010 \\
0.2 & 0.193895101008 & 0.193895121487 & 0.466457257512 & 0.466457257479 \\
0.5 & 0.466457257480 & 0.466457282978 & 0.724228025534 & 0.724228025484 \\
0.8 & 0.724228025488 & 0.724228067006 & 0.890857244698 & 0.890857245264 \\
1.0 & 0.890857245275 & 0.890856966173 & & \\
\hline
\end{tabular}

TABLE 4: Comparison of the absolute error functions $\left|e_{1, N}(x)\right|,\left|e_{2, N}(x)\right|$ for $N=5,6,8,11$ of problem (52).

\begin{tabular}{lcccc}
\hline & & \multicolumn{2}{c}{ Lucas collocation method } & $\left|e_{1,8}\left(x_{i}\right)\right|$ \\
$x_{i}$ & $\left|e_{1,5}\left(x_{i}\right)\right|$ & $\left|e_{1,6}\left(x_{i}\right)\right|$ & $4.9550 e-11$ & $2.3912 e-12$ \\
\hline 0.1 & $5.9187 e-7$ & $3.0339 e-8$ & $4.9303 e-11$ & $2.8583 e-12$ \\
0.2 & $1.0110 e-6$ & $4.1070 e-8$ & $6.4840 e-11$ & $4.8214 e-12$ \\
0.5 & $1.0978 e-6$ & $5.1093 e-8$ & $9.1255 e-11$ & $8.8867 e-12$ \\
0.8 & $9.0094 e-7$ & $8.3187 e-8$ & $1.1536 e-9$ & $1.4366 e-11$ \\
1.0 & $1.3659 e-5$ & $5.6070 e-7$ & $\left|e_{2,8}\left(x_{i}\right)\right|$ & $1.5621 e-12$ \\
\hline$x_{i}$ & $\left|e_{2,5}\left(x_{i}\right)\right|$ & $\left|e_{2,6}\left(x_{i}\right)\right|$ & $2.4764 e-11$ & $1.5043 e-12$ \\
0.1 & $2.9327 e-7$ & $1.5123 e-8$ & $2.4641 e-11$ & $2.5725 e-12$ \\
0.2 & $5.0134 e-7$ & $2.0479 e-8$ & $3.2407 e-11$ & $6.7968 e-12$ \\
0.5 & $5.4596 e-7$ & $2.5498 e-8$ & $4.5610 e-11$ & $1.3288 e-11$ \\
0.8 & $4.5116 e-7$ & $4.1518 e-8$ & $5.7653 e-10$ & \\
1.0 & $6.7555 e-6$ & $2.7910 e-7$ &
\end{tabular}


TABLE 5: Comparison of the absolute error functions $\left|e_{1, N}(x)\right|$ and $\left|e_{2, N}(x)\right|$ for different methods of problem (52).

\begin{tabular}{|c|c|c|c|c|}
\hline$x_{i}$ & $\begin{array}{c}\text { Chebyshev method in [12] } \\
\qquad\left|e_{1,5}\left(x_{i}\right)\right|\end{array}$ & Stehfest Method in [29] & $\begin{array}{l}\text { Present method } \\
\qquad\left|e_{1,5}\left(x_{i}\right)\right|\end{array}$ & $\begin{array}{l}\text { Present method } \\
\qquad\left|e_{1,11}\left(x_{i}\right)\right| \\
\end{array}$ \\
\hline 0.1 & $4.5105 e-5$ & $6.7614 e-5$ & $5.9187 e-7$ & $2.3912 e-12$ \\
\hline 0.2 & $7.9850 e-5$ & $8.4949 e-5$ & $1.0110 e-6$ & $2.8583 e-12$ \\
\hline 0.5 & $9.7191 e-5$ & $3.1897 e-3$ & $1.0978 e-6$ & $4.8214 e-12$ \\
\hline 0.8 & $8.0060 e-5$ & $5.2028 e-3$ & $9.0094 e-7$ & $8.8867 e-12$ \\
\hline 1.0 & $1.0677 e-4$ & $1.1938 e-2$ & $1.3659 e-5$ & $1.4366 e-11$ \\
\hline$x_{i}$ & $\begin{array}{c}\text { Chebyshev method in [12] } \\
\qquad\left|e_{2,5}\left(x_{i}\right)\right|\end{array}$ & Stehfest Method in [29] & $\begin{array}{l}\text { Present method } \\
\qquad\left|e_{2,5}\left(x_{i}\right)\right|\end{array}$ & $\begin{array}{l}\text { Present method } \\
\qquad\left|e_{2,11}\left(x_{i}\right)\right|\end{array}$ \\
\hline 0.1 & $2.2477 e-5$ & $8.4086 e-6$ & $2.9327 e-7$ & $1.5621 e-12$ \\
\hline 0.2 & $3.9847 e-5$ & $1.9575 e-5$ & $5.0134 e-7$ & $1.5043 e-12$ \\
\hline 0.5 & $4.8907 e-5$ & $2.2420 e-4$ & $5.4596 e-7$ & $2.5725 e-12$ \\
\hline 0.8 & $4.0642 e-5$ & $4.6470 e-4$ & $4.5116 e-7$ & $6.7968 e-12$ \\
\hline 1.0 & $5.3904 e-5$ & $4.7100 e-4$ & $6.7555 e-6$ & $1.3288 e-11$ \\
\hline
\end{tabular}

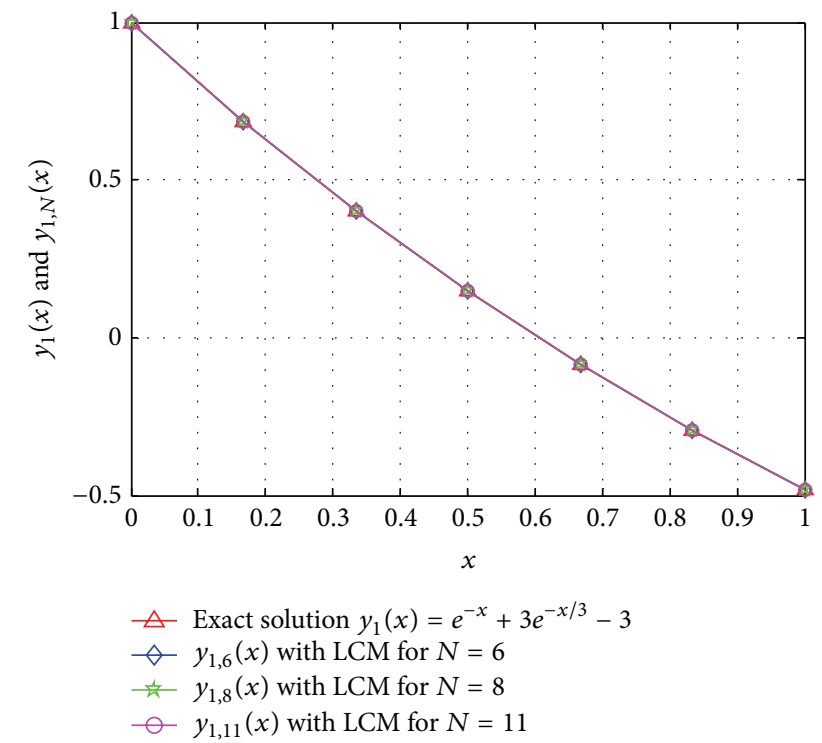

(a)

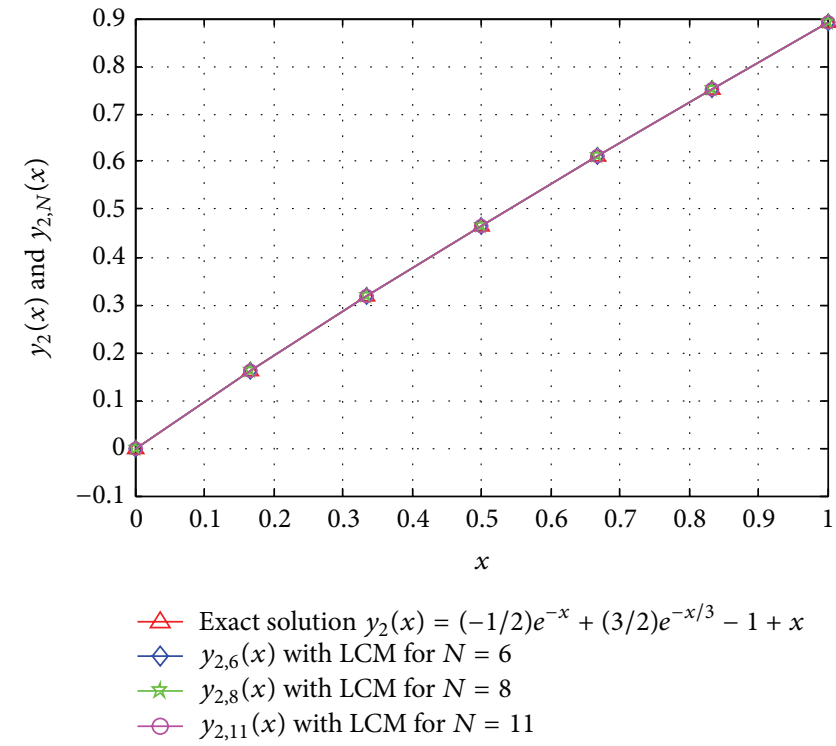

(b)

Figure 3: (a) Comparison of the exact solution $y_{1}(x)$ and the approximate solutions $y_{1, N}(x)$. (b) Comparison of the exact solution $y_{2}(x)$ and the approximate solutions $y_{2, N}(x)$.

Example 2. Let us consider the linear differential equations system given by

$$
\begin{array}{r}
y_{1}^{(1)}(x)+y_{2}^{(1)}(x)+y_{2}(x)=x-e^{-x}, \\
y_{1}^{(1)}(x)+4 y_{2}^{(1)}(x)+y_{1}(x)=1+2 e^{-x}, \\
0 \leq x \leq 1
\end{array}
$$

with the initial conditions

$$
y_{1}(0)=1, \quad y_{2}(0)=0
$$

which has the exact solutions $y_{1}(x)=e^{-x}+3 e^{-x / 3}-3$ and $y_{2}(x)=-e^{-x} / 2+3 e^{-x / 3} / 2-1+x[12,29]$. In this problem $k=2$, $m=1, p_{1,1}^{1}=1, p_{1,1}^{0}=0, p_{1,2}^{1}=1, p_{1,2}^{0}=1, g_{1}(x)=x-e^{-x}$, $p_{2,1}^{1}=1, p_{2,1}^{0}=1, p_{2,2}^{1}=4, p_{2,2}^{0}=0$, and $g_{2}(x)=1+2 e^{-x}$.
We can write the fundamental matrix equation of the problem (52) from (28) as

$$
\left\{\mathbf{P}_{0} \mathbf{X} \overline{\mathrm{D}}+\mathbf{P}_{1} \mathbf{X} \overline{\mathbf{B}} \overline{\mathrm{D}}\right\} \mathbf{A}=\mathbf{G} .
$$

By using our method, the approximate solutions of the problem (52) for $N=6$ are obtained as

$$
\begin{aligned}
y_{1,6}(x)= & -1.999999999999999904 x \\
& +0.6666586621368288521 x^{2} \\
& -0.185113238503384888 x^{3} \\
& +(0.429421011617410024 e-1) x^{4} \\
& -(0.7937368973290355 e-2) x^{5} \\
& +(0.9237777698612969 e-3) x^{6}
\end{aligned}
$$




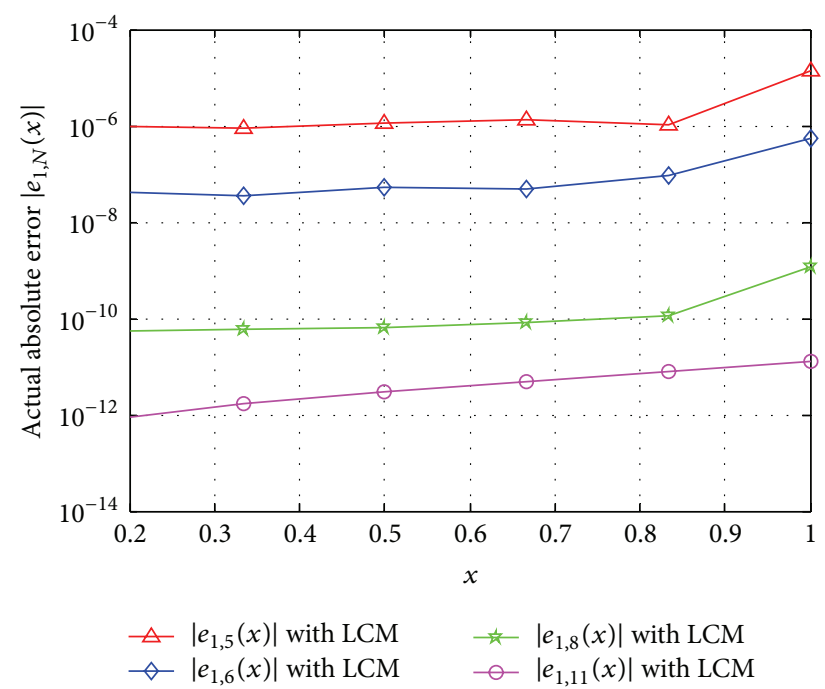

(a)

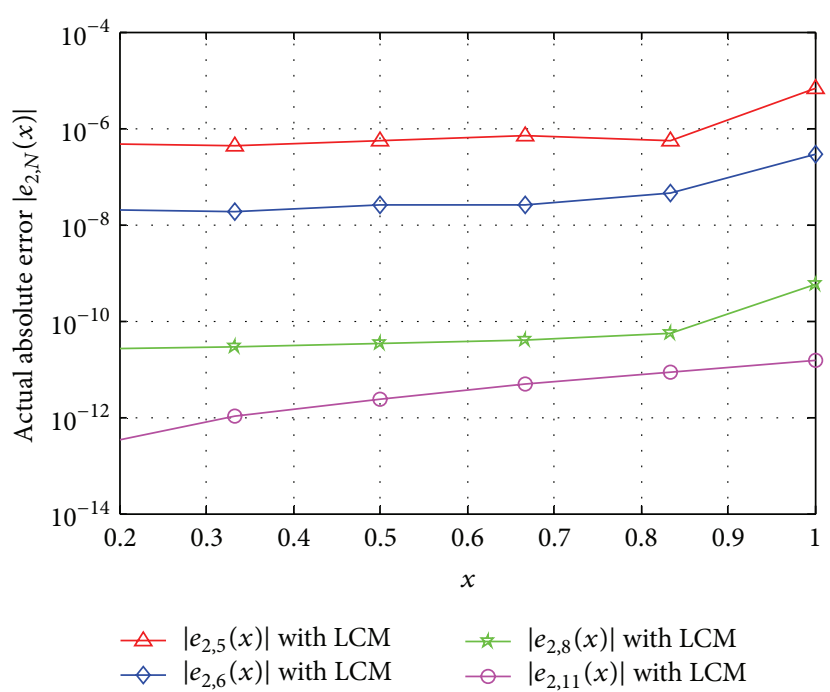

(b)

Figure 4: (a) Comparison of the absolute error functions $\left|e_{1, N}(x)\right|$. (b) Comparison of the absolute error functions $e_{2, N}(x)$.

TABLE 6: Numerical results of the exact solutions and the approximate solutions for $N=2,5,9$ of problem (60).

\begin{tabular}{lcccr}
\hline$x_{i}$ & Exact solution & \multicolumn{3}{c}{ Lucas polynomial solutions } \\
& $y_{1}\left(x_{i}\right)$ & $y_{1,2}\left(x_{i}\right)$ & $y_{1,5}\left(x_{i}\right)$ & 1.105169808728 \\
0.1 & 1.105170918076 & 1.106706136074 & 1.221400910855 & 1.105170918072 \\
0.2 & 1.221402758160 & 1.226824544296 & 1.648719504255 & 1.221402758157 \\
0.5 & 1.648721270700 & 1.667653401853 & 2.225540191066 & 1.648721270696 \\
0.8 & 2.225540928492 & 2.229192708743 & 2.718246576634 & 2.25540928487 \\
1.0 & 2.718281828459 & 2.670613607410 & $y_{2,5}\left(x_{i}\right)$ & 0.099833416647 \\
\hline & $y_{2}\left(x_{i}\right)$ & $y_{2,2}\left(x_{i}\right)$ & 0.099833619019 & 0.198669330795 \\
0.1 & 0.099833416646 & 0.098994748986 & 0.198669599126 & 0.479425538603 \\
0.5 & 0.198669330795 & 0.195978995944 & 0.479425418757 & 0.717356090898 \\
0.8 & 0.479425538604 & 0.474868724650 & 0.717355091659 & 0.841470984845 \\
1.0 & 0.717356090899 & 0.735663935104 & 0.841480130265 & $y_{3,9}\left(x_{i}\right)$ \\
\hline & 0.841470984808 & 0.899474898600 & $y_{3,5}\left(x_{i}\right)$ & 2.100175083352 \\
\hline 0.1 & $y_{3}\left(x_{i}\right)$ & $y_{3,2}\left(x_{i}\right)$ & 2.100174590140 & 2.201469335999 \\
0.2 & 2.100175083354 & 2.101927846772 & 2.201468393096 & 2.526303832587 \\
0.5 & 2.201469336001 & 2.207711387088 & 2.526302577195 & 2.922247637835 \\
0.8 & 2.526303832591 & 2.548196169301 & 2.922246514015 & 3.258584134265 \\
1.0 & 2.922247637840 & 2.923382193410 & 3.258568370866 & \\
\hline
\end{tabular}

+1.0000000000000000808 ,

$y_{2,6}(x)=1.000000000000000105 x$

$-0.1666626772075632049 x^{2}$

$+(0.74038219115823775 e-1) x^{3}$

$-(0.199282827658208166 e-1) x^{4}$

$+(0.3866617014883018 e-2) x^{5}$

$-(0.4569099838690821 e-3) x^{6}$

$+(0.108 e-16)$.
In order to calculate the corrected Lucas polynomial solutions, let us consider the error problem

$$
\begin{aligned}
& e_{1,6}^{(1)}(x)+e_{2,6}^{(1)}(x)+e_{2,6}(x)=-R_{1,6}(x), \\
& e_{1,6}^{(1)}(x)+4 e_{2,6}^{(1)}(x)+e_{1,6}(x)=-R_{2,6}(x)
\end{aligned}
$$

such that $e_{1,6}(0)=0, e_{2,6}(0)=0$, and the residual functions are

$$
\begin{gathered}
R_{1,6}(x)=y_{1,6}^{(1)}(x)+y_{2,6}^{(1)}(x)+y_{2,6}(x)-x+e^{-x} \\
R_{2,6}(x)=y_{1,6}^{(1)}(x)+4 y_{2,6}^{(1)}(x)+y_{1,6}(x)-1-2 e^{-x} .
\end{gathered}
$$


TABLE 7: Comparison of the actual absolute errors $\left|e_{1, N}(x)\right|,\left|e_{2, N}(x)\right|$ for $N=2,5,9$ of problem (60).

\begin{tabular}{lccc}
\hline$x_{i}$ & $\left|e_{1,2}\left(x_{i}\right)\right|$ & $\left|e_{1,5}\left(x_{i}\right)\right|$ & $\left|e_{1,9}\left(x_{i}\right)\right|$ \\
\hline 0.1 & $1.5352 e-3$ & $1.1093 e-6$ & $3.5633 e-12$ \\
0.2 & $5.4218 e-3$ & $1.8473 e-6$ & $3.1738 e-12$ \\
0.5 & $1.8932 e-2$ & $1.7664 e-6$ & $4.0266 e-12$ \\
0.8 & $3.6518 e-3$ & $7.3743 e-7$ & $5.3975 e-12$ \\
1.0 & $4.7668 e-2$ & $3.5252 e-5$ & $1.3694 e-10$ \\
\hline$x_{i}$ & $\left|e_{2,2}\left(x_{i}\right)\right|$ & $\left|e_{2,5}\left(x_{i}\right)\right|$ & $\left|e_{2,9}\left(x_{i}\right)\right|$ \\
\hline 0.1 & $8.3867 e-4$ & $2.0237 e-7$ & $6.1484 e-13$ \\
0.2 & $2.6903 e-3$ & $2.6833 e-7$ & $2.8246 e-13$ \\
0.5 & $4.5568 e-3$ & $1.1985 e-7$ & $4.6422 e-13$ \\
0.8 & $1.8308 e-2$ & $9.9924 e-7$ & $1.5204 e-12$ \\
1.0 & $5.8004 e-2$ & $9.1455 e-6$ & $3.7266 e-11$ \\
\hline$x_{i}$ & $\left|e_{3,2}\left(x_{i}\right)\right|$ & $\left|e_{3,5}\left(x_{i}\right)\right|$ & $\left|e_{3,9}\left(x_{i}\right)\right|$ \\
\hline 0.1 & $1.7528 e-3$ & $4.9321 e-7$ & $1.7336 e-12$ \\
0.2 & $6.2421 e-3$ & $9.4291 e-7$ & $1.8815 e-12$ \\
0.5 & $2.1892 e-2$ & $1.2554 e-6$ & $3.0301 e-12$ \\
0.8 & $1.1346 e-3$ & $1.1238 e-6$ & $4.3129 e-12$ \\
1.0 & $6.5799 e-2$ & $1.5763 e-5$ & $6.2478 e-11$ \\
\hline
\end{tabular}

TABLE 8: Comparison of the errors of the methods for problem (60).

\begin{tabular}{lcc}
\hline$x_{i}$ & $\begin{array}{c}\text { Differential transform } \\
\text { method in [2] }\end{array}$ & Present method $\left|e_{1,9}\left(x_{i}\right)\right|$ \\
\hline 0.1 & $2.0092 e-11$ & $3.5633 e-12$ \\
0.2 & $2.6046 e-9$ & $3.1738 e-12$ \\
0.5 & $1.6526 e-6$ & $4.0266 e-12$ \\
0.8 & $4.6173 e-5$ & $5.3975 e-12$ \\
1.0 & $2.2627 e-4$ & $1.3694 e-10$ \\
\hline$x_{i}$ & Differential transform & Present method $\left|e_{2,9}\left(x_{i}\right)\right|$ \\
\hline 0.1 & method in [2] & $6.1484 e-13$ \\
0.2 & $1.9839 e-11$ & $2.8246 e-13$ \\
0.5 & $2.5383 e-9$ & $4.6422 e-13$ \\
0.8 & $1.5447 e-6$ & $1.5204 e-12$ \\
1.0 & $4.1242 e-5$ & $3.7266 e-11$ \\
\hline$x_{i}$ & $1.9568 e-4$ & Present method $\left|e_{3,9}\left(x_{i}\right)\right|$ \\
\hline 0.1 & Differential transform & $1.7336 e-12$ \\
0.2 & $2.0340 e-11$ & $1.8815 e-12$ \\
0.5 & $2.6681 e-9$ & $3.0301 e-12$ \\
0.8 & $1.7493 e-6$ & $4.3129 e-12$ \\
1.0 & $5.0305 e-5$ & $6.2478 e-11$ \\
\hline & $2.5080 e-4$ &
\end{tabular}

By solving the error problem (56) for $M=8$, the estimated Lucas error functions $e_{1,6,8}(x)$ and $e_{2,6,8}(x)$ to $e_{1,6}(x)$ and $e_{2,6}(x)$ are obtained as

$$
\begin{aligned}
e_{1,6,8}(x)= & -(0.1210 e-17) x \\
& -(0.178885917374467780 e-3) x^{7} \\
& +(0.142216618722969108 e-4) x^{8}
\end{aligned}
$$

$$
\begin{aligned}
& +(0.79326892394531528 e-5) x^{2} \\
& -(0.71083092941970440 e-4) x^{3} \\
& +(0.2633108714690733660 e-3) x^{4} \\
& -(0.486294856240169128 e-3) x^{5} \\
& +(0.4502363215270527764 e-3) x^{6} \\
& -(0.6384 e-18) \\
e_{2,6,8}(x)= & (0.6913 e-18) x \\
& +(0.892342831286434209 e-4) x^{7} \\
& -(0.710875043057030262 e-5) x^{8} \\
& -(0.39530091035351939 e-5) x^{2} \\
& +(0.354285130160153836 e-4) x^{3} \\
& -(0.1312587470335659604 e-3) x^{4} \\
& +(0.2424513686179994193 e-3) x^{5} \\
& -(0.22451354772782758896 e-3) x^{6} \\
& -(0.18124 e-18)
\end{aligned}
$$

Thus, we can calculate the corrected Lucas polynomial solutions $y_{1,6,8}(x)$ and $y_{2,6,8}(x)$ as

$$
\begin{aligned}
y_{1,6,8}(x)= & -2 x+0.6666665948 x^{2} \\
& -0.1851843216 x^{3}+(0.4320541203 e-1) x^{4} \\
& -(0.8423663829 e-2) x^{5} \\
& +(0.1374014091 e-2) x^{6}+1 \\
& -(0.178885917374467780 e-3) x^{7} \\
& +(0.142216618722969108 e-4) x^{8},
\end{aligned}
$$




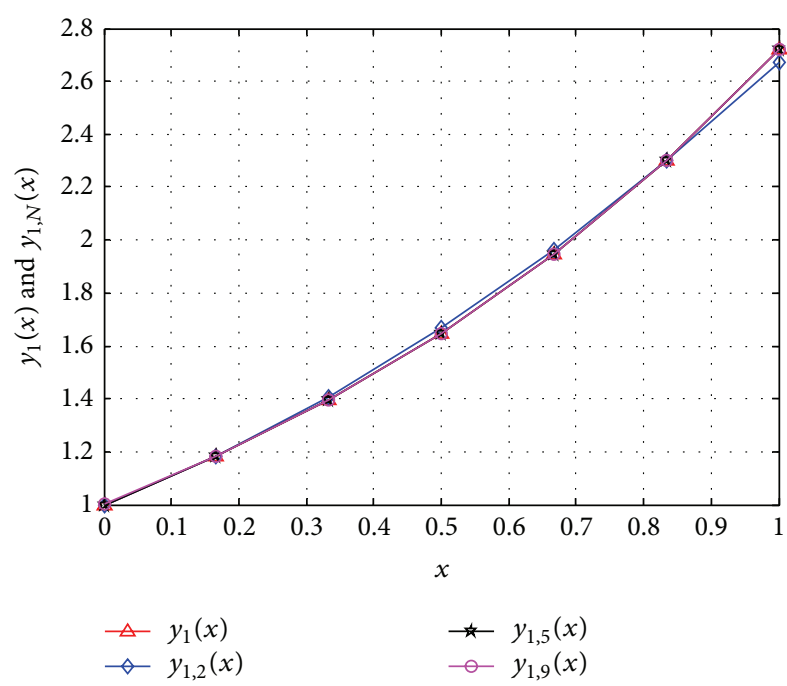

(a)

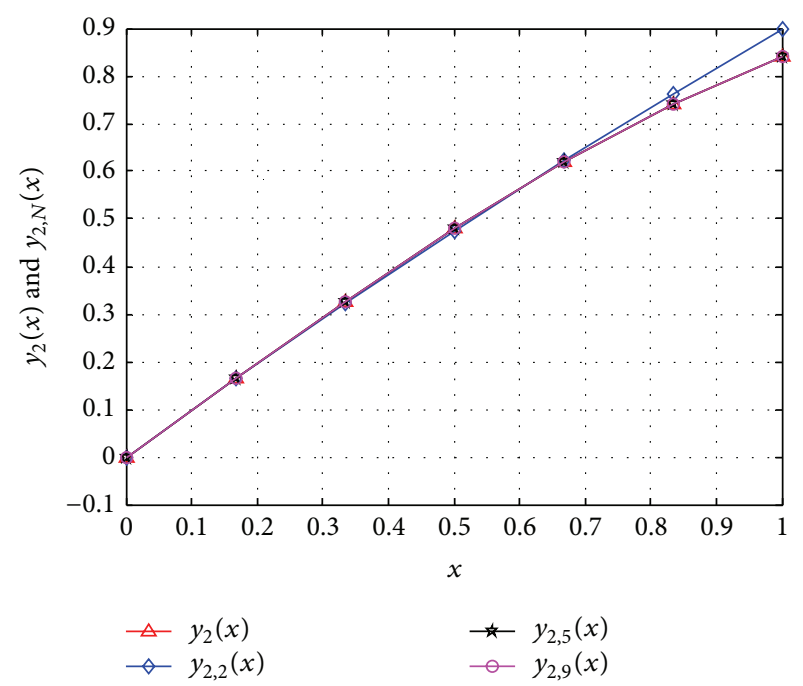

(b)

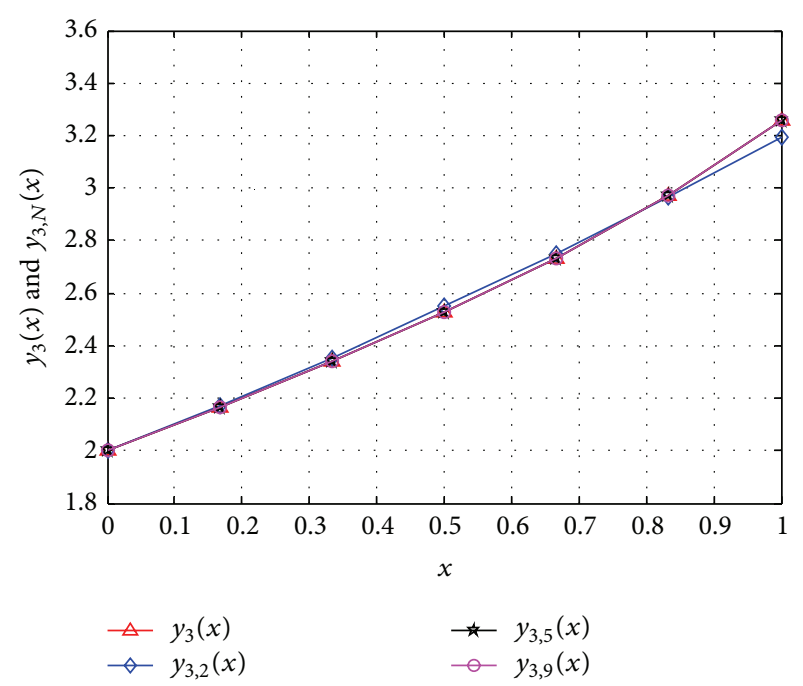

(c)

Figure 5: (a) Comparison of the exact solution $y_{1}(x)$ and the approximate solutions $y_{1, N}(x)$. (b) Comparison of the exact solution $y_{2}(x)$ and the approximate solutions $y_{2, N}(x)$. (c) Comparison of the exact solution $y_{3}(x)$ and the approximate solutions $y_{3, N}(x)$.

$$
\begin{aligned}
y_{2,6,8}(x)= & x-0.1666666302 x^{2} \\
& +(0.7407364763 e-1) x^{3} \\
& -(0.2005954152 e-1) x^{4} \\
& +(0.4109068384 e-2) x^{5} \\
& -(0.6814235316 e-3) x^{6}+(0.1061876 e-16) \\
& +(0.892342831286434209 e-4) x^{7} \\
& -(0.710875043057030262 e-5) x^{8} .
\end{aligned}
$$

It is seen from Table 1 and Figures 1(a) and 1(b) that the accuracy of solution increases when the values of $N$ and $M$ increase.

Table 2 and Figures 2(a) and 2(b) display that the actual and estimated errors are very close to zero and almost identical.

Table 3 and Figures 3(a) and 3(b) show that when the value of $N$ increases, the accuracy of solution increases.

Table 4 and Figures 4(a) and 4(b) show that the value of $N$ is increased; the actual absolute errors decrease rapidly.

In addition, this problem was solved by Akyüz-Daşoğlu and Sezer [12] and Davies and Crann [29]. Now, let us compare our method (LCM) with the other methods (Chebyshev method and Stehfest method) given by [12, 29]. Table 5 indicates this comparison. 


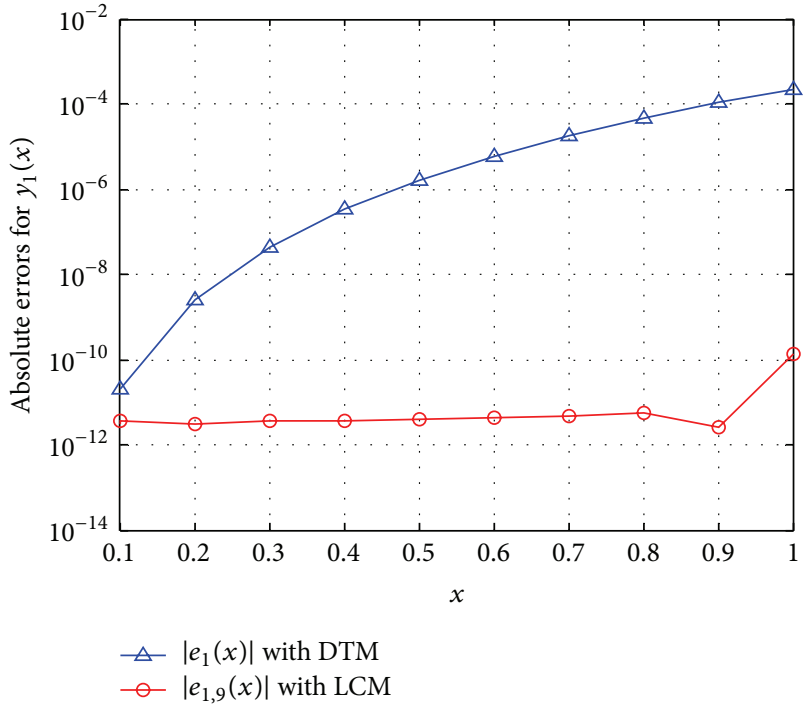

(a)

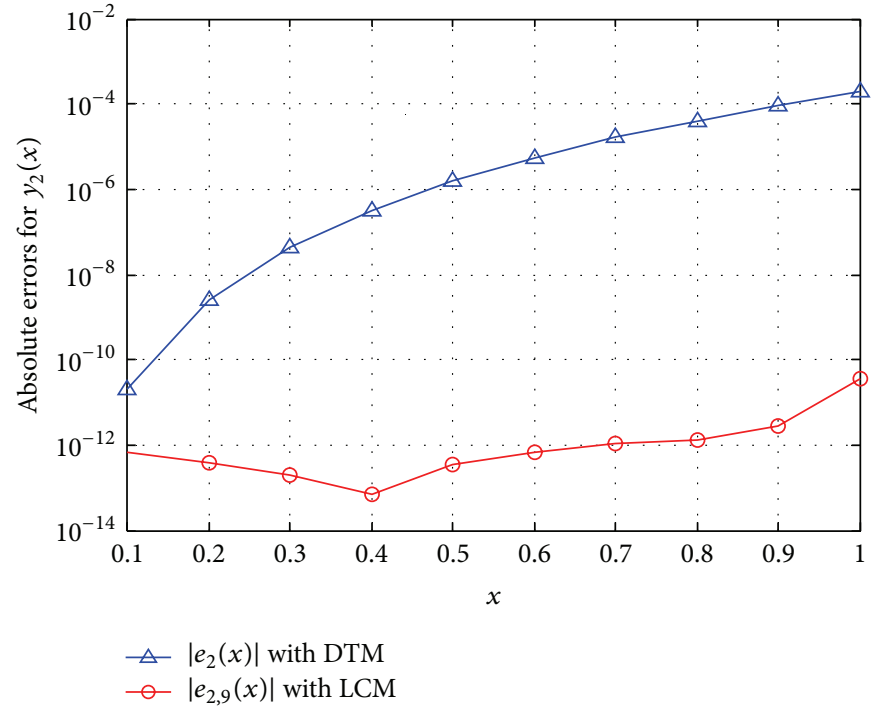

(b)

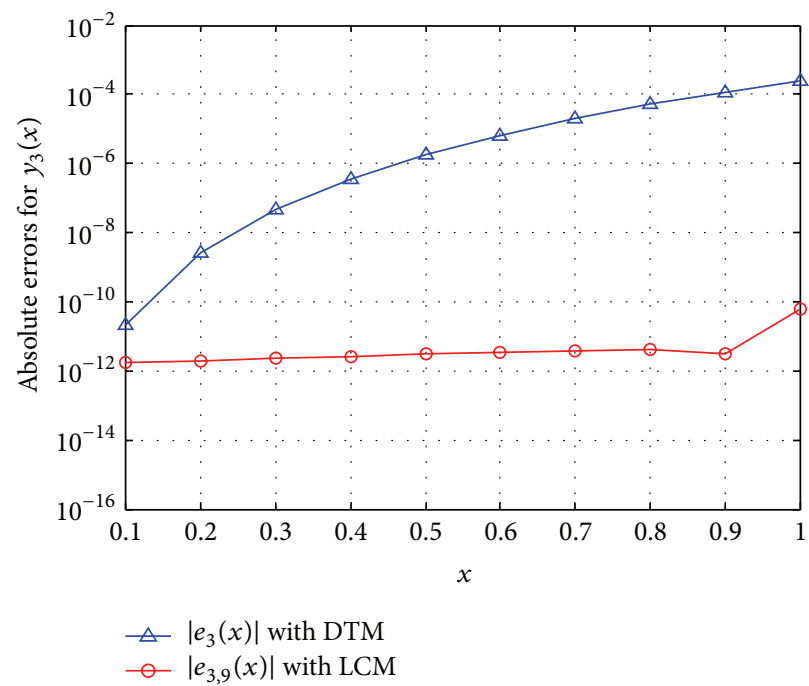

(c)

Figure 6: (a) Comparison of the absolute error functions for $y_{1}(x)$. (b) Comparison of the absolute error functions for $y_{2}(x)$. (c) Comparison of the absolute error functions for $y_{3}(x)$.

It is seen from Table 5 that the present method gives better approximations than the other methods given by [12, 29].

Example 3. Let us consider the linear differential equations system given by

$$
\begin{array}{r}
y_{1}^{(1)}(x)-y_{3}(x)=-\cos (x), \\
y_{2}^{(1)}(x)-y_{3}(x)=-e^{x}, \\
y_{3}^{(1)}(x)-y_{1}(x)+y_{2}(x)=0, \\
0 \leq x \leq 1
\end{array}
$$

with the initial conditions

$$
y_{1}(0)=1, \quad y_{2}(0)=0, \quad y_{3}(0)=2
$$

which has the exact solutions $y_{1}(x)=e^{x}, y_{2}(x)=\sin (x)$, and $y_{3}(x)=e^{x}+\cos (x)$ [2]. By using the method, the approximate solutions of the problem (60) for $N=2,5$ are obtained as

$$
\begin{gathered}
y_{1,2}(x)=1+x+0.67061360741023699830 x^{2}, \\
y_{2,2}(x)=-(0.1 e-18)+x-0.1005251013995184324 x^{2}, \\
y_{3,2}(x)=2+x+0.19278467720243885767 x^{2}, \\
y_{1,5}(x)=0.999999999999999999824 \\
+1.0000000000000000125 x \\
+0.49976858483072423438 x^{2} \\
+0.16822545527123026922 x^{3}
\end{gathered}
$$




$$
\begin{aligned}
& +(0.3772109990725761363 e-1) x^{4} \\
& +(0.12531436624829093170 e-1) x^{5} \\
y_{2,5}(x)= & +1.000000000000000009 x \\
+ & (0.45327625709752 e-4) x^{2} \\
- & 0.166988949339599508 x^{3} \\
+ & (0.782824607296818 e-3) x^{4} \\
+ & (0.7640927371818304 e-2) x^{5}+(0.6 e-17), \\
y_{3,5}(x)= & 1.99999999999999999978 \\
& +1.0000000000000000004 x \\
& -(0.938977776578073 e-4) x^{2} \\
& +0.1672264869687396031 x^{3} \\
& +(0.820919229141536686 e-1) x^{4} \\
& +(0.93438587611964760 e-2) x^{5} .
\end{aligned}
$$

It is seen from Table 6 and Figures 5(a), 5(b), and 5(c) that the accuracy increases as the $N$ increase.

Table 7 shows that while the value of $N$ is increased, the errors decrease rapidly. Now, we compare the present method with the differential transform method given by [2].

It is seen from Table 8 that the present method (LCM) is very effective compared to the differential transform method (DTM) for problem (60).

Figures 6(a), 6(b), and 6(c) display the actual absolute error functions obtained by present method for $N=9$ and the differential transform method. These figures display that the results gained by the present method are better than those obtained by the differential transform method.

\section{Conclusions}

It is known that solving the high-order linear differential equations system is usually very difficult analytically. In this case, it is required to approximate solutions. In this paper, a new method based on the Lucas polynomials with the help of the residual error function for solving system of high-order linear differential equations numerically is presented. When the obtained results are investigated in examples, it can be seen that the developed method is very effective compared to the others. Also, it can be seen from the tables and the figures that the accuracy increased when the value of $N$ is increased. The approximate solutions are obtained in a short time with computer programmes such as Maple, Mathematica, and Matlab. We have used the Maple and Matlab for computations and graphics, respectively. Additionally, the presented method can be applied to the other system of linear integral and integrodifferential equations.

\section{Conflict of Interests}

The authors declare that there is no conflict of interests regarding the publication of this paper.

\section{References}

[1] M. Tatari and M. Dehghan, "Improvement of He's variational iteration method for solving systems of differential equations," Computers \& Mathematics with Applications, vol. 58, no. 11-12, pp. 2160-2166, 2009.

[2] M. Thongmoon and S. Pusjuso, "The numerical solutions of differential transform method and the Laplace transform method for a system of differential equations," Nonlinear Analysis: Hybrid Systems, vol. 4, no. 3, pp. 425-431, 2010.

[3] I. H. Abdel-Halim Hassan, "Application to differential transformation method for solving systems of differential equations," Applied Mathematical Modelling, vol. 32, no. 12, pp. 2552-2559, 2008.

[4] A. Kurnaz and G. Oturanç, "The differential transform approximation for the system of ordinary differential equations," International Journal of Computer Mathematics, vol. 82, no. 6, pp. 709-719, 2005.

[5] F. Ayaz, "Solutions of the system of differential equations by differential transform method," Applied Mathematics and Computation, vol. 147, no. 2, pp. 547-567, 2004.

[6] H. Jafari and V. Daftardar-Gejji, "Revised Adomian decomposition method for solving systems of ordinary and fractional differential equations," Applied Mathematics and Computation, vol. 181, no. 1, pp. 598-608, 2006.

[7] J. Biazar, E. Babolian, and R. Islam, "Solution of the system of ordinary differential equations by Adomian decomposition method," Applied Mathematics and Computation, vol. 147, no. 3, pp. 713-719, 2004.

[8] F. M. Mahomed, I. Naeem, and A. Qadir, "Conditional linearizability criteria for a system of third-order ordinary differential equations," Nonlinear Analysis: Real World Applications, vol. 10, no. 6, pp. 3404-3412, 2009.

[9] C. W. Soh and F. M. Mahomed, "Linearization criteria for a system of second-order ordinary differential equations," International Journal of Non-Linear Mechanics, vol. 36, no. 4, pp. 671677, 2001.

[10] A. Saadatmandi and J. A. Farsangi, "Chebyshev finite difference method for a nonlinear system of second-order boundary value problems," Applied Mathematics and Computation, vol. 192, no. 2, pp. 586-591, 2007.

[11] M. Dehghan, M. Shakourifar, and A. Hamidi, "The solution of linear and nonlinear systems of Volterra functional equations using Adomian-Pade technique," Chaos, Solitons \& Fractals, vol. 39, no. 5, pp. 2509-2521, 2009.

[12] A. Akyüz-Daşoğlu and M. Sezer, "Chebyshev polynomial solutions of systems of high-order linear differential equations with variable coefficients," Applied Mathematics and Computation, vol. 144, no. 2-3, pp. 237-247, 2003.

[13] O. R. Işık, Z. Güney, and M. Sezer, "Bernstein series solutions of pantograph equations using polynomial interpolation," Journal of Difference Equations and Applications, vol. 18, no. 3, pp. 357374, 2012.

[14] S. Yalçınbaş, M. Aynıgül, and M. Sezer, "A collocation method using Hermite polynomials for approximate solution of pantograph equations," Journal of the Franklin Institute, vol. 348, no. 6, pp. 1128-1139, 2011. 
[15] Y. Öztürk and M. Gülsu, "A numerical approach for solving initial-boundary value problem describing the process of cooling of a semi-infinite body by radiation," Applied Mathematical Modelling, vol. 37, no. 5, pp. 2709-2716, 2013.

[16] N. Bildik, A. Konuralp, and S. Yalçinbaş, "Comparison of Legendre polynomial approximation and variational iteration method for the solutions of general linear Fredholm integro-differential equations," Computers and Mathematics with Applications, vol. 59, no. 6, pp. 1909-1917, 2010.

[17] Ş. Yüzbaşı, "Laguerre approach for solving pantograph-type Volterra integro-differential equations," Applied Mathematics and Computation, vol. 232, pp. 1183-1199, 2014.

[18] Ş. Yüzbaşı, "An efficient algorithm for solving multi-pantograph equation systems," Computers \& Mathematics with Applications, vol. 64, no. 4, pp. 589-603, 2012.

[19] Y. Çenesiz, Y. Keskin, and A. Kurnaz, "The solution of the Bagley-Torvik equation with the generalized Taylor collocation method," Journal of the Franklin Institute, vol. 347, no. 2, pp. $452-$ 466, 2010.

[20] K. Maleknejad and Y. Mahmoudi, "Taylor polynomial solution of high-order nonlinear Volterra-Fredholm integro-differential equations," Applied Mathematics and Computation, vol. 145, no. 2-3, pp. 641-653, 2003.

[21] F. A. Oliveira, "Collocation and residual correction," Numerische Mathematik, vol. 36, no. 1, pp. 27-31, 1980.

[22] İ. Çelik, "Approximate calculation of eigenvalues with the method of weighted residuals-collocation method," Applied Mathematics and Computation, vol. 160, no. 2, pp. 401-410, 2005.

[23] İ. Çelik, "Collocation method and residual correction using CHEbyshev series," Applied Mathematics and Computation, vol. 174, no. 2, pp. 910-920, 2006.

[24] S. Shahmorad, "Numerical solution of the general form linear Fredholm-Volterra integro-differential equations by the Tau method with an error estimation," Applied Mathematics and Computation, vol. 167, no. 2, pp. 1418-1429, 2005.

[25] Ş. Yüzbaşı and M. Sezer, "An improved Bessel collocation method with a residual error function to solve a class of LaneEmden differential equations," Mathematical and Computer Modelling, vol. 57, no. 5-6, pp. 1298-1311, 2013.

[26] A. Constandache, A. Das, and F. Toppan, "Lucas polynomials and a standard Lax representation for the polytropic gas dynamics," Letters in Mathematical Physics, vol. 60, no. 3, pp. 197-209, 2002.

[27] P. Filipponi and A. F. Horadam, "Second derivative sequences of Fibonacci and Lucas polynomials," The Fibonacci Quarterly, vol. 31, no. 3, pp. 194-204, 1993.

[28] T. Koshy, Fibonacci and Lucas Numbers with Applications, A Wiley-Interscience Publication, New York, NY, USA, 2001.

[29] A. Davies and D. Crann, "The solution of differential equations using numerical Laplace transforms," International Journal of Mathematical Education in Science and Technology, vol. 30, no. 1, pp. 65-79, 1999.

[30] A. Saadatmandi, M. Dehghan, and A. Eftekhari, "Application of He's homotopy perturbation method for non-linear system of second-order boundary value problems," Nonlinear Analysis. Real World Applications, vol. 10, no. 3, pp. 1912-1922, 2009. 


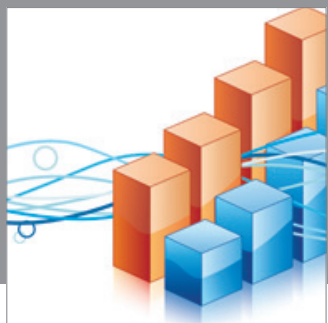

Advances in

Operations Research

mansans

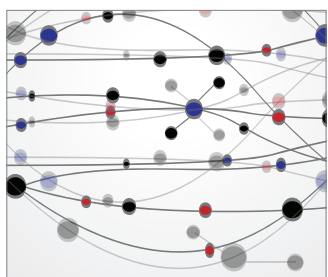

The Scientific World Journal
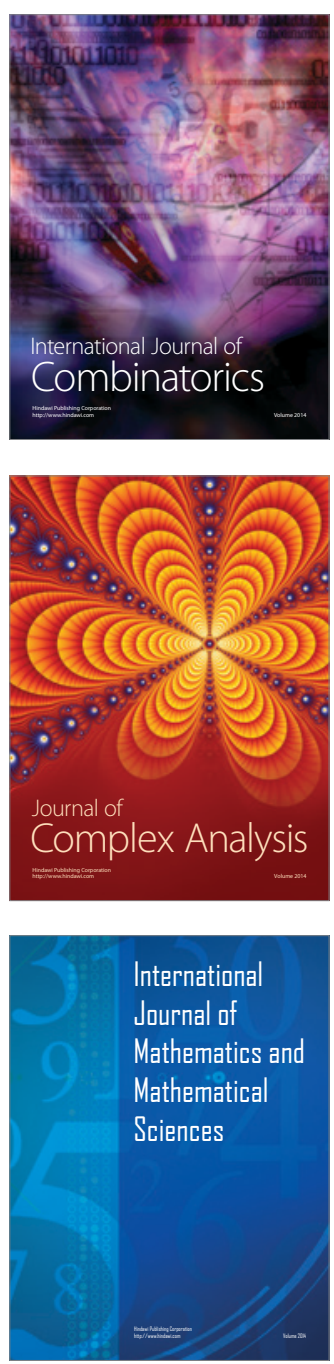
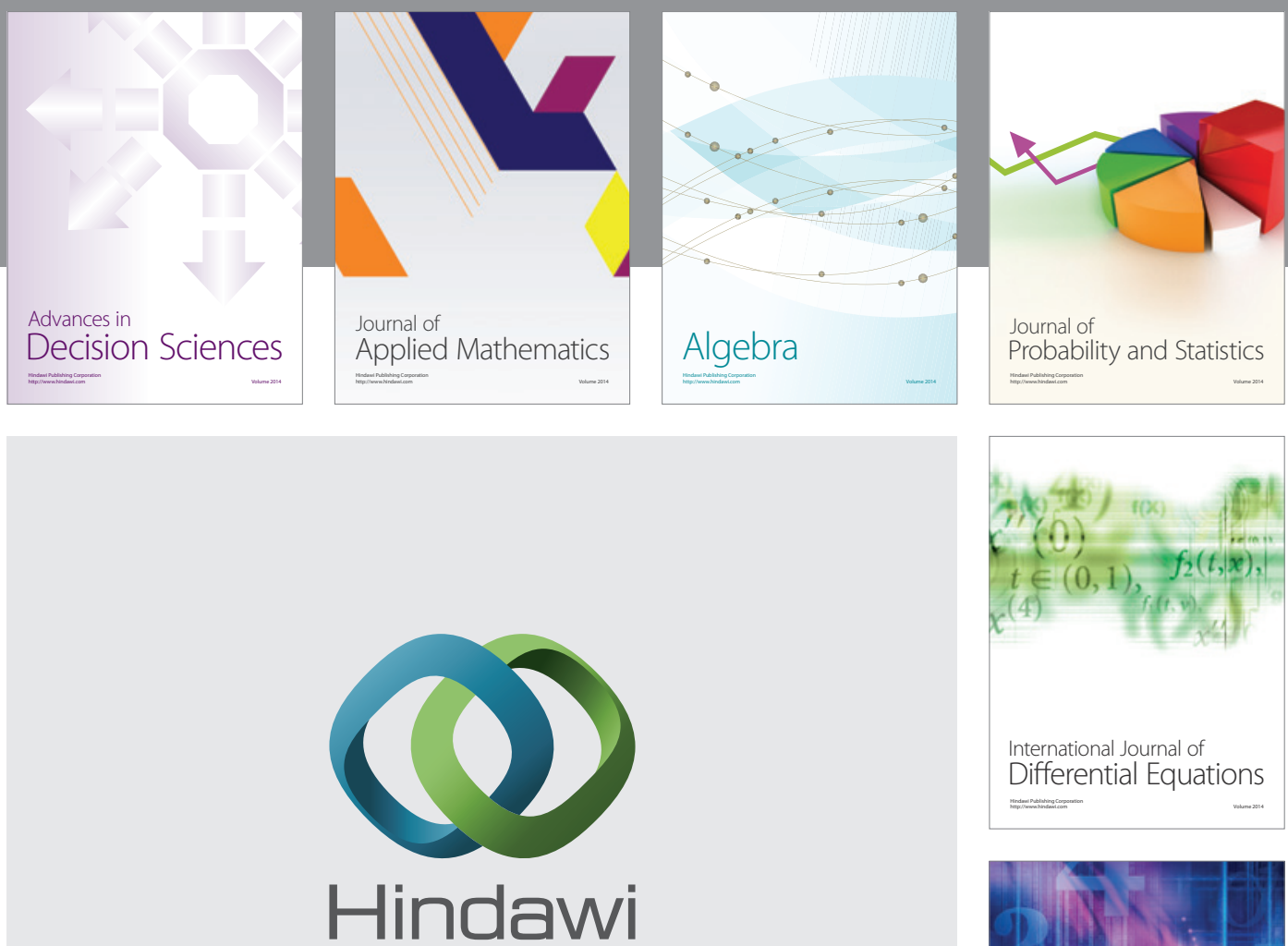

Submit your manuscripts at http://www.hindawi.com
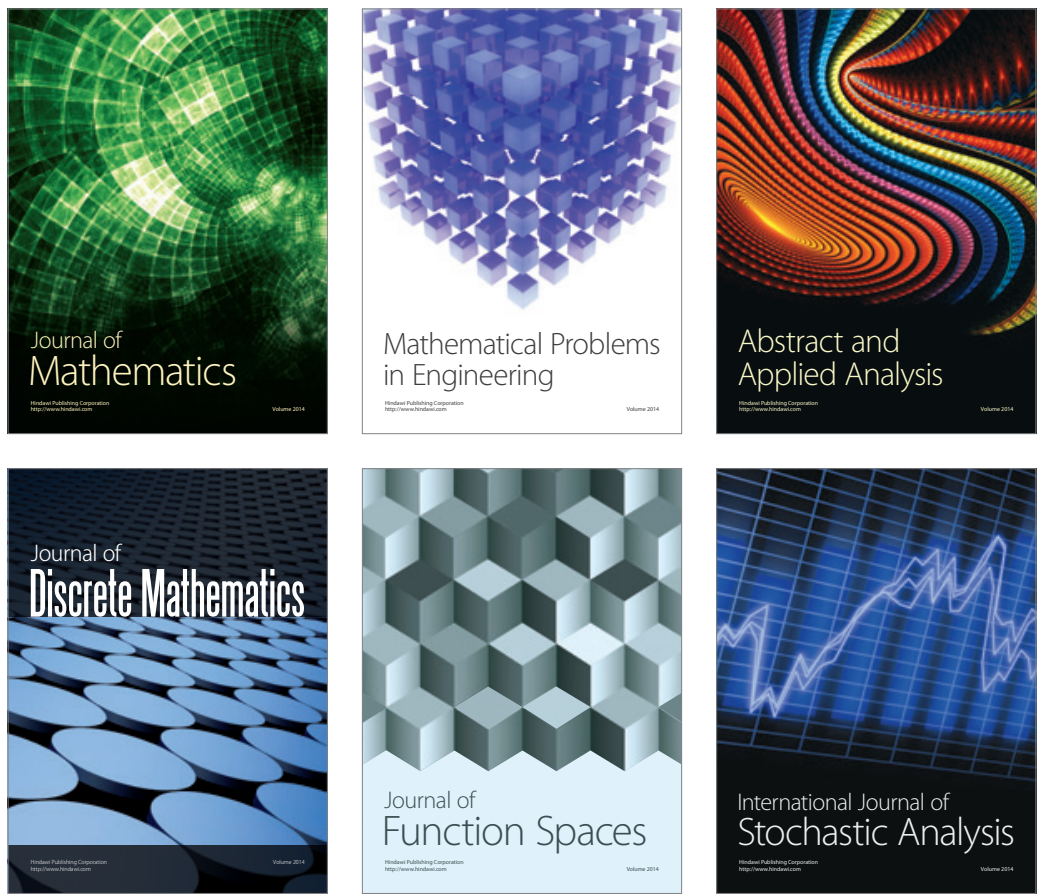

Journal of

Function Spaces

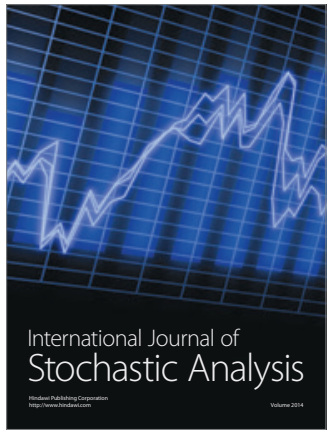

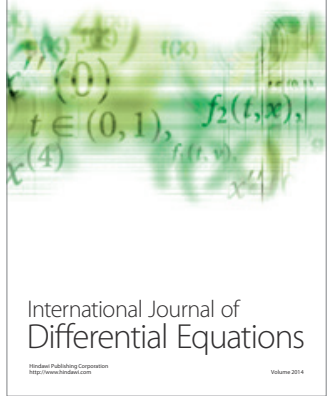
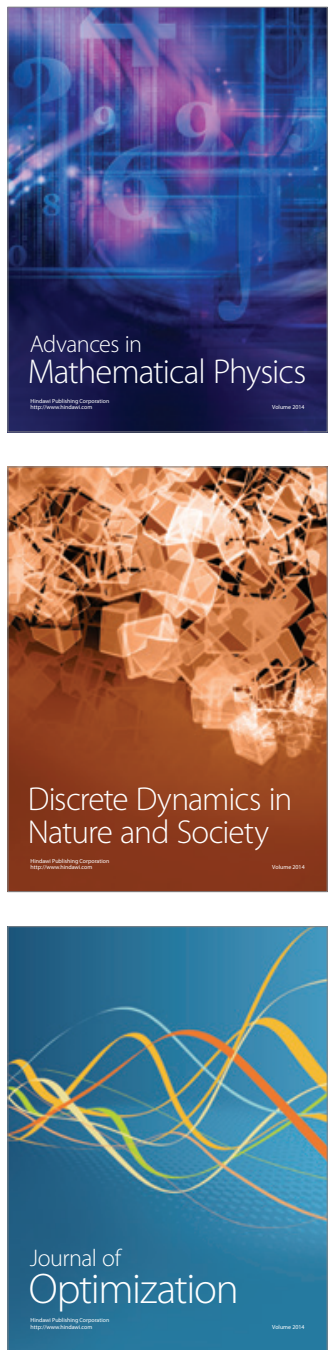